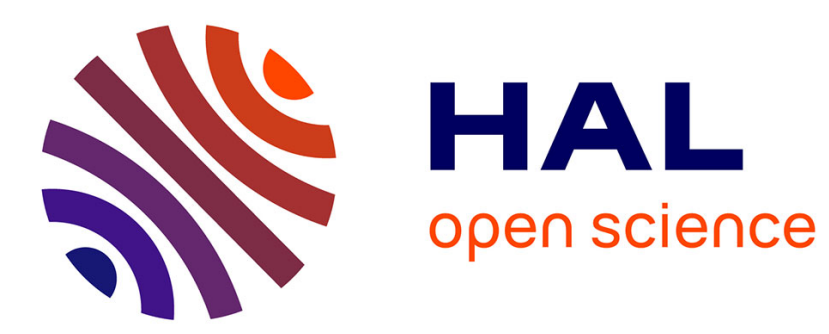

\title{
Temperature profiles in filamentary dielectric barrier discharges at atmospheric pressure
}

\author{
N Jidenko, E Bourgeois, J-P Borra
}

\section{To cite this version:}

N Jidenko, E Bourgeois, J-P Borra. Temperature profiles in filamentary dielectric barrier discharges at atmospheric pressure. Journal of Physics D: Applied Physics, 2010, 43 (29), pp.295203. 10.1088/0022$3727 / 43 / 29 / 295203$. hal-00569657

\section{HAL Id: hal-00569657 https://hal.science/hal-00569657}

Submitted on 25 Feb 2011

HAL is a multi-disciplinary open access archive for the deposit and dissemination of scientific research documents, whether they are published or not. The documents may come from teaching and research institutions in France or abroad, or from public or private research centers.
L'archive ouverte pluridisciplinaire HAL, est destinée au dépôt et à la diffusion de documents scientifiques de niveau recherche, publiés ou non, émanant des établissements d'enseignement et de recherche français ou étrangers, des laboratoires publics ou privés. 


\title{
Temperature profiles in filamentary Dielectric Barrier Discharges at atmospheric pressure
}

\author{
N Jidenko, E Bourgeois and J-P Borra \\ Laboratoire de Physique des Gaz et des Plasmas (UMR 8578 CNRS - Univ Paris-Sud \\ Orsay, F-91405) SUPELEC, Plateau Moulon, F-91192 Gif-Sur-Yvette, France
}

\begin{abstract}
The physico-chemical properties of atmospheric pressure filamentary Dielectric Barrier Discharge (f-DBD) depend on its electrical characteristics and thermal profile. In this paper, a method for separating thermal and electrical effects is developed. Therefore, thermal profiles of f-DBD are studied for well defined electrical characteristics of filaments: all filaments are quasi identical with a controlled spatio-temporal density. The temperatures of gas, dielectric surface and plasma depend on the surface density and the temporal frequency of the filaments and can be altered by modifying the heat transfer. Different methods to control these temperatures are depicted. Moreover, heat transfer through conduction and convection from dielectric surface is shown to be the dominant heating mechanism for the flowing gas in the reactor. Finally, experimental results show that the temperature gradient around filaments can be controlled by the frequency of the applied voltage. Actually, the temperature difference between the filament and surrounding gas is independent of the applied frequency below 10 $\mathrm{kHz}$. However, above $10 \mathrm{kHz}$, it increases linearly with the frequency. At high frequency, the time between two successive filaments occurring at the same position becomes smaller than the relaxation time constant of the thermal exchanges $(\sim 0.1 \mathrm{~ms})$. Thus, this rise in temperature can be attributed to inefficient heat transfer from the filament formation zone.
\end{abstract}

\section{Introduction}

Reactive and ionising properties of non-equilibrium plasmas at atmospheric pressure have been investigated for oxidizing properties, for pollution abatement, surface treatments, as well as for aerosol charging and production [1-5]. The AC polarisation of millimetre plane-toplane Dielectric Barrier Discharges (DBD) in air at atmospheric pressure mostly leads to similar thin and brief discharge filaments [6-8]. In filamentary Dielectric Barrier Discharge (fDBD), the temperature profiles of the heated volumes of FD and surfaces are critical for the transport of primary species (diffusion, thermophoresis) and for the kinetics of chemical reactions and of nucleation [9-12].

Despite moderate gas heating (below $500 \mathrm{~K}$ ), the composition and the concentration of stable chemical species and aerosol particles produced by f-DBD depend on the thermal profile of the discharge gap. In this article, the term "thermal profile" refers to the reactor temperatures (plasma, gas and surface), thermal gradients and their (temperature and thermal gradients) evolution in time. The periodic evolution of local temperature profiles around filaments controls the thermal diffusion of primary species and the kinetics of local surface and volume reactions. Thus, the temperature profile partly controls the unitary production of chemicals per filament and the related total production of the reactor [10, 13, 14].

For instance, ozone production is related to both energy density (ratio of input power and gas flow rate in $\mathrm{J}^{-1}$ ) and dielectric surface temperature. Ozone decomposition is favoured at high surface temperatures by two main mechanisms i) surface reactions and ii) reactions with NOx formed at high temperatures (> $350 \mathrm{~K}$ ) [1]. The decomposition by NOx is known as discharge poisoning and was first reported in 1860 [15].

This study is focused on the control of thermal profile in f-DBD. The two main objectives are : 
i) to evaluate the main thermal processes and to identify the main operating parameters controlling the temperature profiles,

ii) to show that the temperature as well as the temperature gradient can be partly decoupled from electrical parameters.

This study is part of a broader experimental approach that aims to identify the relative influence of temperature and temperature gradient on the chemical and physical processes (e.g. ozone production/destruction kinetics, nucleation of condensable gaseous species, aerosol charging dynamics, aerosol kinematics...) involved in f-DBD [16, 17].

Numerous studies deal with rotational temperature measurements of the gas inside the discharge filaments, heat exchange and energy balance in DBD. Whatever the initial gas temperature is, the temperature rise in the filaments in $\mathrm{Ar}, \mathrm{H}_{2} \mathrm{O}$ mixtures (i.e. the temperature difference between the neutral species in the filaments and the surrounding gas) is constant of about $50 \mathrm{~K}$ [18].

Methane fed DBD has recently been investigated with a particular focus on the energy balance and it appears that over $50 \%$ of the input power is ultimately converted into heat through the dielectric barriers $[19,20]$; for a $0.5 \mathrm{~mm}$ gap length, the temperature rise in the filaments is constant of about $100 \mathrm{~K}$ whatever the input power and the gas flow velocity are [19]. Similar results have been obtained in He and air, with a cylindrical arrangement. The fraction of the input power that is dissipated as heat highly depends on the gas composition and decreases with increasing input power [21].

Models have highlighted that temperature rise in the filaments depends on the energy injection in the filament and on the dynamic of energy dissipation, including the changes in enthalpy due to chemical reactions and radiative decay of excited states [22]. The temperature rise in a filament depends on the energy of filament and is not constant along the filament. It varies from $5-25 \mathrm{~K}$ in the middle of filament to a maximum temperature of 200$800 \mathrm{~K}$ on the cathode for $\varepsilon_{\mathrm{r}}=5-50$ [21, 22]. Moreover for a $2 \mathrm{~mm}$ gap length with $\varepsilon_{\mathrm{r}}=5$, the mean temperature rise due to joule heating is about $27 \mathrm{~K}$, after $0.34 \mu$ s there is an additional temperature rise of $23 \mathrm{~K}$, due to excited states of $\mathrm{N}_{2}$ dissipating their energy into translational modes [22].

Using smooth parallel dielectric surfaces in a given DBD arrangement, the electrical characteristics of filamentary discharges are the same within $25 \%$ whatever the voltage and the frequency between 1 and $60 \mathrm{kHz}$ are [23]. Under such defined electrical conditions, the control of heat exchange allows the modification of the temperature at constant electrical parameters. The dielectric surface and outgoing gas temperatures are measured to evaluate the thermal exchanges between filaments, surrounding gas and dielectric surfaces. The temperature and thermal gradient around filaments are evaluated through emission spectroscopy of the second positive system of nitrogen.

\section{Experimental setup}

Two similar dielectric barrier discharge arrangements with 0.5 and $1 \mathrm{~mm}$ gap widths are compared. The dielectric material is alumina. Brass electrodes $3 \mathrm{~cm}$ long and $1 \mathrm{~cm}$ wide were placed on opposite sides of the two parallel plates of alumina (roughness $<0.08 \mu \mathrm{m}$, thickness $\left.\mathrm{e}=0.55 \mathrm{~mm}, \varepsilon_{\mathrm{r}} \sim 9\right)$. The electrodes are designed to minimize edge effects. A silicon paste surrounding the electrodes prevented the formation of arcs and other parasite discharges outside the gap. Moreover, the DBD arrangements are conditioned between two half casings made of stumatite (aluminosilicate mineral) to control heat exchange with the environment.

A high sinusoidal AC voltage supplies the plasma reactors. The frequency was tuneable between 1 and $60 \mathrm{kHz}$, with a maximum peak-to-peak voltage of $21 \mathrm{kV}$. Current and voltage waveforms were stored with an oscilloscope for data-processing as described in [23] to define 
the input power, the number of filaments per surface and time unit and the electrical characteristics of the filaments (charge per pulse, maximum of the pulse current, duration).

The flow rate of dry air (relative humidity $<5 \%$ ) was controlled with mass flow controllers between 1 and $6 \mathrm{~L} \cdot \mathrm{min}^{-1}$. The maximum mean gas velocity (gap width $1 \mathrm{~mm}$ ) was $10 \mathrm{~m} \cdot \mathrm{s}^{-1}$ and the residence time in the DBD was between 3 and $18 \mathrm{~ms}$.



Figure 1: Experimental setup for electrical and thermal characterizations.

Optical emission spectroscopy of the second positive system of nitrogen $\left(C^{3} \Pi_{u} \rightarrow B^{3} \Pi_{g}\right)$ was performed to evaluate the kinetic temperature of neutral molecules in the filament (hereafter referred as plasma temperature, at $+/-30 \mathrm{~K}$ in the range $300-3000 \mathrm{~K}$ ) as described in [24]. The rotational temperature of the excited state $\mathrm{C}^{3} \Pi_{\mathrm{u}}$ is determined by comparison of experimentally measured spectra with simulated spectra of the second positive system of nitrogen. The theoretical spectra are calculated from the analysis detailed in [25]. Rotational and translation temperatures can differ. Nevertheless, at high pressure, low energy collision transfer is efficient and relaxations are fast enough for the equilibrium to be completely established at the photon emission, molecules in the rotational states and neutral gas molecules are thus in equilibrium. Hence, the rotational temperature of the excited state $C^{3} \Pi_{u}$, corresponds to the temperature of neutral species in the plasma [24, 26]. The light is collected with an optical fibre in a cone of $12.5^{\circ}$ acceptance angle for 30 seconds. As a result, the collected light is an average in time and space of the emission of several filaments. Moreover, the filament temperature is overestimated due to the collection of the light from the spots situated at the filament bases which are hotter than the filament. Nevertheless, we assume that the evolution of this measured temperature is representative of the evolution of filament temperature.

The average (in time and space) temperature of the dielectric surface (Ts) was evaluated by the measurement of electrode temperature (Tel) with a K-type thermocouple. Simulation results show that the average dielectric surface and the electrode temperature are similar within a few Kelvin at thermal quasi-equilibrium state. Calculation have been conducted assuming stationary and uniform heat transfer to the dielectric surfaces and taking into account heat conduction through dielectric plates, electrodes and casing as well as radiative and convective transfers from casing to ambient air,

The temperature of the outgoing gas $(\mathrm{Tg})$ was measured with a K-type thermocouple downstream a copper tube $(3 \mathrm{~cm}$ long with $10 \mathrm{~mm}$ inner diameter leading to transit time from 140 to $840 \mathrm{~ms}$ ). 


\section{Results and discussion}

One route to develop aerosol processes by atmospheric pressure electrical discharges is based on the control of physical and chemical mechanisms by electro-thermal decoupling. As the discharge filaments are quasi identical, macroscopic measurements downstream of the DBD (e.g. ozone concentration, aerosol concentration, aerosol mean charge...) can be used to study local mechanisms occurring around one filament. Macroscopic measurement is related to the cumulative effect of the local contributions of different filaments, this contribution should be weighted by a factor depending on the evolution and the mixing along the reactor. The influence of temperature on each mechanism can thus be investigated by electro-thermal decoupling i.e. with (i) different temperature profiles at constant electrical characteristics of the plasma or (ii) similar temperature profile with different charges per filament or (iii) similar temperature profile and charge per filament with different distributions of filaments in time and space. The electro-thermal decoupling thus implies the independent control of the characteristics of quasi-identical filaments, of their distribution in time and space, and of the temperature profiles through heat exchange at constant electrical parameters.

The electrical characterisation of DBD has already been presented in [23, 26]. The frequency of the applied voltage controls the repetition rate of the filament at the same place whereas the applied voltage controls the spacing between filaments. This paper focuses on thermal control and electro-thermal decoupling of filamentary-DBD at constant electrical characteristics of filaments through temperature measurements. One objective of this paper is to help users to dimension the DBD reactor to reach the temperature ranges required by the application (e.g. catalysis, soot combustion, self cleaned filter...).

The first section aims at defining the operating parameter ranges for which filaments are similar. Then, gas and surface temperature measurements are presented versus the number of quasi identical filamentary discharges. Different methods to decouple dielectric surface temperature from electrical parameters are presented. Finally, the temperature of neutral species in the filament and the local temperature gradient around the filament are estimated though optical emission spectroscopy measurements. The objective is to find a way to control the transport of chemical species and aerosol particles through the thermal profile tuned by frequency.

\subsection{Similarity of discharge filaments}

To define the operating parameter ranges (applied voltage, voltage frequency, gas flow rate and surface temperature) leading to quasi-identical filaments, a statistical analysis of the charge per current pulse related to filamentary discharge has been conducted.

The term filament refers here to a filamentary discharge also called microdischarge which develops by the streamer mechanism. The reader should be careful that another definition can be encountered: successive streamers, macroscopically observed as a bright spatially localized filament [27]. For a gap of $1 \mathrm{~mm}$, filament lasts $20 \mathrm{~ns}$ with typical current amplitude of 20 $\mathrm{mA}$. Filaments are distributed in space but deposited charges on dielectric surfaces on both sides of the filament leads to the formation of a new filament in the axis of the charge spot, every time the polarity changed. The energy per filament is a critical characteristic to study the dynamic of energy injection in the reactor. The energy per filament can be considered as proportional to the charge per filament [28]. The factor of proportionality depends on the nature of the gas and the discharge arrangement.

The frequency distributions of charge per current pulse at $1 \mathrm{kHz}$ (input power $\mathrm{P}=1 \mathrm{~W}$ and electrode temperature $\mathrm{Tel}=25 \mathrm{~K})$ and $60 \mathrm{kHz}(\mathrm{P}=20 \mathrm{Watt}, \mathrm{Tel}=150 \mathrm{~K})$ for a peak-to-peak voltage $\left(\mathrm{V}_{\mathrm{pp}}\right)$ of $17.2 \mathrm{kV}$ are plotted in Figure 2 (a). The evolution of the mean charge per filament with the applied peak-to-peak voltage is depicted for the $1 \mathrm{~mm}$ gap DBD, at $60 \mathrm{kHz}$ in Figure 2 (b). 
(a)

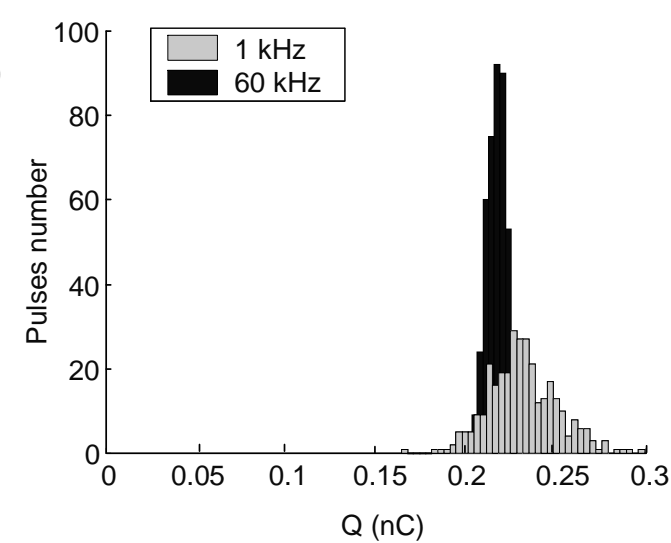

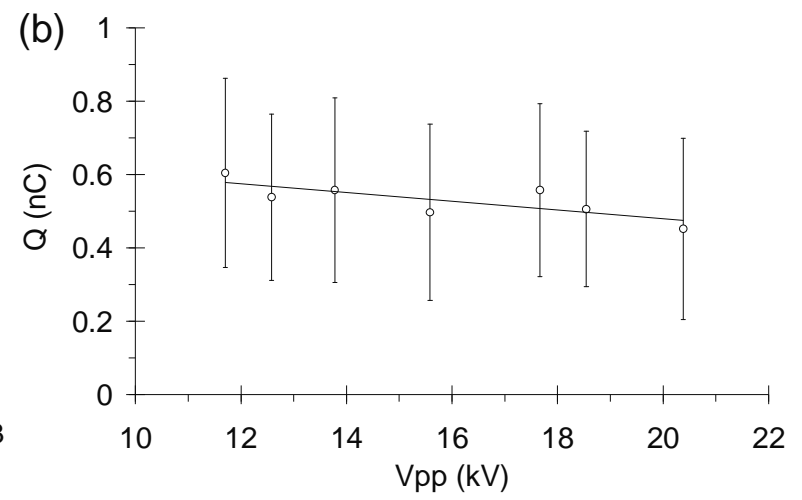

Figure 2: (a) Frequency distributions of charge per filament at $1 \mathrm{kHz}(\mathrm{P}=1 \mathrm{Watt}, \mathrm{Tel}=25 \mathrm{~K})$ and $60 \mathrm{kHz}$ $(\mathrm{P}=20$ Watts, Tel=150 K) for the $0.5 \mathrm{~mm}$ gap length $\mathrm{DBD}$ at $4 \mathrm{lpm}$ (b) Mean charge per filament versus applied peak-to-peak voltage, the error bars correspond to standard deviation, $1 \mathrm{~mm}$ gap, at $60 \mathrm{kHz}$ and $1 \mathrm{lpm}$.

For a given operating condition, all the filaments are similar within $25 \%$ which validates the experimental approach used.

At voltage frequency of $1 \mathrm{kHz}$ and $60 \mathrm{kHz}$, the average charge per pulse is 0.23 and $0.22 \mathrm{nC}$ with standard deviation of 0.06 and 0.003 respectively (cf. Figure $2 \mathrm{a}$ ).

Varying the applied voltage, from 11 to $20 \mathrm{kV}$, leads to a decrease of the mean charge per filament by $25 \%$ (cf. Figure $2 \mathrm{~b}$ ). The filaments lay charges on both dielectric surfaces which reduces the electric field strength to below the extinction field until the next half period. During the next half period, the charges deposited on both dielectric surfaces increase the field strength until the next filament develops at the same place. Decreasing the inter-filament distance results in a shielding effect from surface charges of adjacent filaments which reduces the quantity of charge per filament [29]. For a given geometry, the number of microdischarges per half period, distributed in time and space on the total surface of the plane-to-plane DBD, depends almost entirely on the applied voltage; the voltage frequency only plays a minor role [29]. At first sight, one can consider that filaments occur at the same location. Thus, the evolution of the electric field profile is repeated at the frequency as the applied voltage. Planeto-plane DBD leads to the reproduction of quasi identical filament with a controlled number per time and surface unit with respect to voltage and frequency.

Changing the gas flow rate from 1 to $6 \mathrm{lpm}$ results in a $14 \%$ increase of the mean charge per filament $(0.18 \mathrm{nC}$ to $0.21 \mathrm{nC})$ with a reduction of the dispersion (standard deviation from 14.5 to $5.7 \mathrm{pC}$ ). This effect is probably due to the fact that the volume in which the filament occurs is better purged the higher the gas flow rate is. Indeed remaining active species produced by the preceding filament, occurring at the same place, modify the physical processes such as ionisation and attachment for the formation of a new filament.

The variation of average gas temperature, from 300 to $420 \mathrm{~K}$, leads to the decrease of the mean charge per filament within less than $5 \%$. Thus, the reduction of the average charge per filament with the increase of the applied voltage frequency, observed in Figure 2, can be partly attributed to thermal effects. Many parameters driving plasma electrical properties are affected by gas and surface temperature (gas density, surface conductivity of dielectric material, secondary emission...).

Gas temperature affects the gas density which in turn modifies reduced electric field (ratio between electric field and gas density) and hence the related electron energy distribution. Nevertheless, for different temperatures, electrical pulses are quasi identical. The similarity of current pulses proves that filaments occur for identical reduced electric field. This is confirmed by the linear reduction in the applied onset voltage for filament initiation, with the increase in temperature [30]. For instance, at $21 \mathrm{kV}$ peak-to-peak voltage, the first filament in 
positive polarity occurs for mean applied voltages of $-2.2 \mathrm{kV}$ and $-4.5 \mathrm{kV}$ at $300 \mathrm{~K}$ and $540 \mathrm{~K}$ respectively.

Moreover, the gas density also modifies the distribution of ion space charge required to fulfil Meek's criterion; the propagation of the filament is thus affected. Surface conductivity can modify the surface charge distribution on dielectric surface and the related electric field profile. Finally, the dielectric surface temperature controls the secondary emission. Nevertheless, the electrical characteristics of the filament (charge per filament, reduced electric field profile and electron energy distribution function) can be considered as similar.

To summarize, due to the retro control of the electric field by dielectric surface polarization, filaments can be considered as similar to within $25 \%$ (based on charge per filament), whatever the frequency (from 1 to $60 \mathrm{kHz}$ ), the applied voltage (from 11 to $20 \mathrm{kV}$ ), the average dielectric surface temperature (from 300 to $470 \mathrm{~K}$ ) and the gas flow rate (from 1 to $6 \mathrm{lpm})$ are.

The energy per filament depends on the gap length, the specific capacitance of the dielectric material $\left(\varepsilon_{\mathrm{r}} / \mathrm{e}\right.$ with $\varepsilon_{\mathrm{r}}$ the relative permittivity of the dielectric material and $\mathrm{e}$ the thickness of the dielectric material), the composition and the temperature of the gas. The number of filaments per period depends on the electrode area, applied voltage, gap length and specific dielectric capacitance. Finally, the input power is the product of the energy per filament, the number of filaments per period and the frequency. Operating parameter ranges leading to similar filaments are now defined and thermal exchanges can be investigated.

\subsection{Gas and surface temperatures versus the number of similar Filamentary Discharges} per time and surface unit

Each filamentary discharge is a source of heat, transferring parts of its energy to dielectric surfaces and flowing gas. The dielectric surface temperature depends on the balance between heat sources (input power, area of the electrodes) and heat sinks (shape and mass of the electrodes and of the casing and gas flow regime as well as ambient temperature).

Heat transfer from the plasma to the surface relies on radiative, conductive and convective transfers of charged and neutral species. Moreover, ion neutralization, metastable energy transfer and exothermic chemical reactions on the surface contribute to surface heating. Finally, the high frequency component of the current induces dielectric losses leading to heating of the dielectric material [31]. The gas is heated by electron elastic collisions in the plasma but this mechanism is not important since electrons are lighter than gas molecules. Exothermic chemical reaction and metastable de-excitation (in particular for vibrationally excited species) also contribute to gas heating along the reactor.

Surfaces are cooled down by (i) the incoming gas flow which is heated by conductive, convective and radiative transfers from dielectric surfaces (ii) conduction in the bulk of dielectric material and subsequent heat transfer to DBD casing and ambient atmosphere by convective transfers.

The electrode temperature (which differs from the mean dielectric surface temperature by only a few degrees, cf. section 2) is plotted, at quasi-steady state, versus input power for the $1 \mathrm{~mm}$ gap DBD with a gas flow rate of $2 \mathrm{lpm}$ in Figure 3 (a) and for the 0.5 and $1 \mathrm{~mm}$ gap DBD at constant flow velocity in Figure 3 (b). 

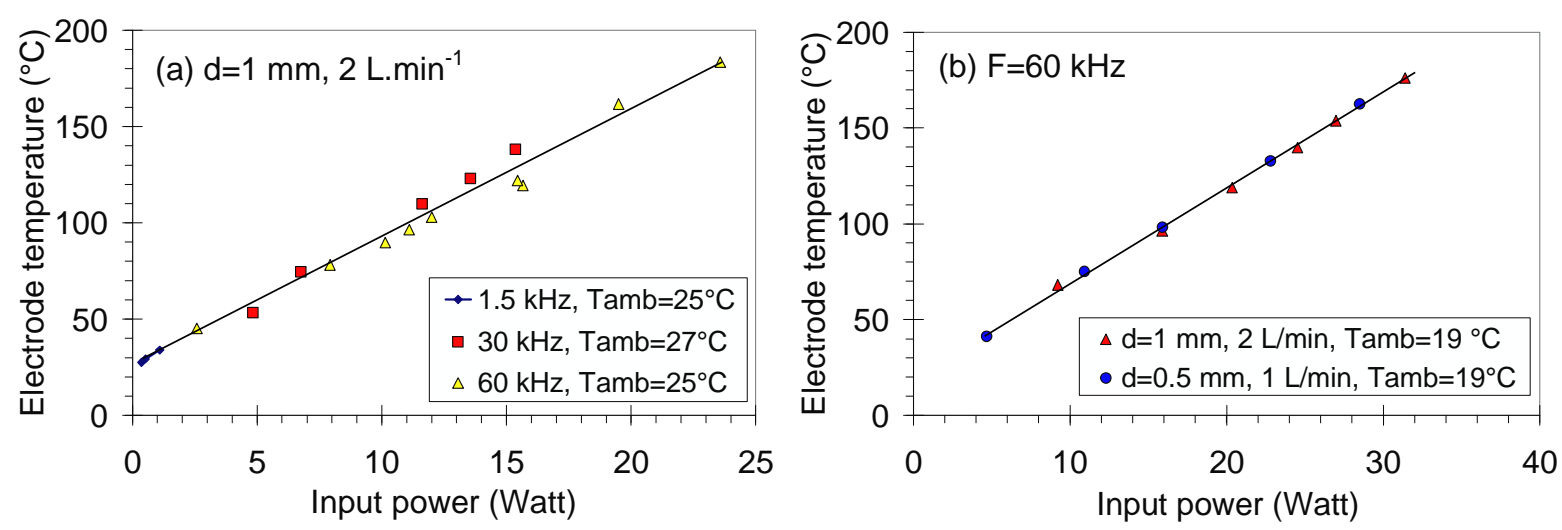

Figure 3: Electrode temperature versus input power (a) at different frequencies of applied voltage 1.5, 30 and $60 \mathrm{kHz}$ (b) for 0.5 and $1 \mathrm{~mm}$ gap length at constant gas flow velocity.

For a given gas flow rate, the electrode temperature linearly increases with the input power (cf. Figure 3 a). The relation between electrode temperature and input power does not change with the voltage frequency, i.e. whatever the distribution of identical filaments in time and space is (cf. section 3.1).

In Figure 3 (b), for different gap lengths ( $1 \mathrm{~mm}$ and $0.5 \mathrm{~mm}$, leading to charge per filament of 0.22 and $0.5 \mathrm{nC}$ respectively) the relation between electrode temperature and input power is the same. Hence, similar transit times and flow regime (Reynolds numbers of 40 and 70 for 0.5 and $1 \mathrm{~mm}$ gap respectively) in the reactors lead to similar heat exchanges. Convective transfers between the dielectric surface and flowing gas seems thus to be dominant as shown hereafter. As a conclusion, the relation between electrode temperature and input power is independent of the dynamics of energy injection (energy per filament and filament distribution in time and space), for a given gas flow rate.

The influence of the gas flow rate on the electrode and gas temperature is plotted in Figure 4 for the $0.5 \mathrm{~mm}$ DBD with an input power of $16 \mathrm{Watts}\left(\mathrm{V}_{\mathrm{pp}}=16 \mathrm{kV}, \mathrm{F}=60 \mathrm{kHz}\right)$.

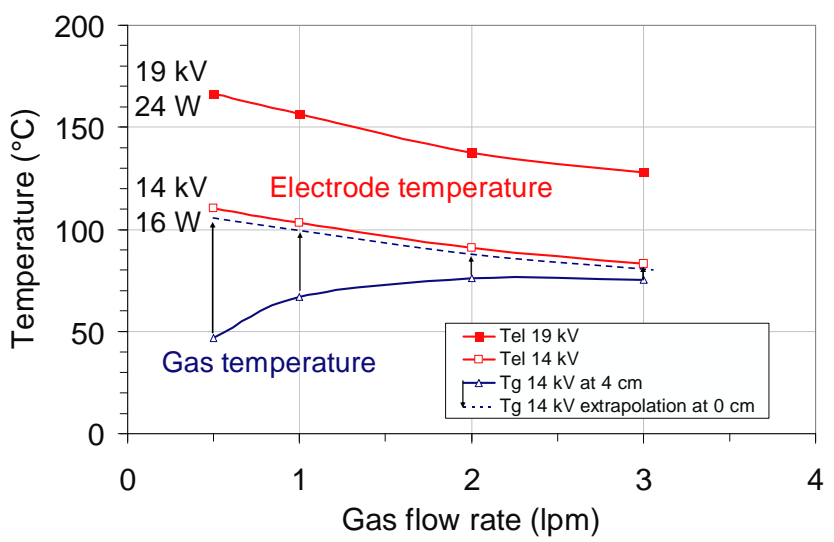

Figure 4: Electrode and gas temperatures versus gas flow rate at $60 \mathrm{kHz}$ in the $0.5 \mathrm{~mm}$ gap DBD.

The reduction of the electrode temperature with the gas flow rate is due to the increase in the heat flux transferred in the flowing gas. Indeed, the enthalpy gained by the feed gas $\left(\mathrm{Q}_{\mathrm{m}} \cdot \mathrm{C}_{\mathrm{p}} \cdot \Delta \mathrm{T}\right)$ increases with increasing gas velocity. Here, $\mathrm{Q}_{\mathrm{m}}$ is the air mass flow rate $\left(\mathrm{kg} . \mathrm{s}^{-1}\right)$, $\mathrm{C}_{\mathrm{p}}$ is the specific heat capacity of air $\left(\mathrm{kg} . \mathrm{s}^{-1}\right)$ and $\Delta \mathrm{T}$ is the gas temperature increase within the DBD reactor.

Figure 4 shows that the gas temperature, at $4 \mathrm{~cm}$ of the DBD exit, presents a maximum versus the flow rate. This maximum depends on the input power or the position of measurement. The copper tube at the exit of the DBD acts as a heat exchanger. At low gas 
flow rate (below $2 \mathrm{lpm}$ ), the residence time in the copper tube is higher leading to lower gas temperature. At high gas flow rate, the reduction of the gas temperature is due to the reduction of the dielectric surface temperature by the gas flow rate. The measured curve is thus an artefact. On approaching the position of measurement to the discharge, the differences between the curves diminish. In laminar flow regime, the average gas temperature at the exit of the discharge is the same as the dielectric surface temperature. Electrode and gas temperatures follow similar evolution with the gas flow rate (cf extrapolation in Figure 4).

Three following arguments support the fact that the convective transfer from hot dielectric surfaces, heated by filament, is the predominant transfer that heats the flowing gas.

The flowing gas is heated by electron-molecule elastic collisions in the filaments, by exothermal volume reactions, by de-excitation of vibrationally excited species. However these processes are less efficient than convective transfer from hot dielectric surfaces. Indeed, the gas temperature downstream the DBD never exceeds the electrode temperature by more than a few Kelvin. This difference can be due either to de-excitation of vibrationally excited species in post discharge or to the fact that the measured temperature is not the temperature of the dielectric surface at the hottest position.

Besides, most of heat transfer in mono DBD with cooled electrodes are directed on the dielectric surface because of plasma propagation on dielectric surfaces [19].

Finally, assuming a filament diameter of 10-100 $\mu \mathrm{m}$ [29], calculations from plasma temperature measurement (cf. section 3.4) show that the average enthalpy produced per time unit by the filament channel is lower than the enthalpy gained by the feed gas. These arguments tend to support convective transfers from dielectric surfaces to the gas as the predominant heating mechanism. Convection at least dominates at the entrance of the reactor where the maximum temperature difference between the gas and the dielectric surface is located.

As shown in Figure 4, the gas flow rate allows the modification of heat exchange and related temperatures with constant electrical parameters (filament characteristics and number of filaments per surface and time unit). The electro-thermal decoupling can also be achieved with other parameters described below.

\subsection{Methods to control surface temperature with constant electrical parameters}

The intensity of heat transfer by conduction, convection and radiation defines the final dielectric surface temperature. Indeed, the relative importance of the different mechanisms of heat exchange can be modified with respect to the flow regime, gas velocity and thermal exchanges around the reactor casing to control DBD surface temperature. For a given set of electrical working conditions and DBD arrangement (applied voltage frequency, electrode area, gas flow rate and external heat exchanges) leading to similar filaments within $5 \%$, table 1 gives examples of electrode temperature variations.

Table 1: Evolution of electrode temperature at constant electrical characteristics of filaments

\begin{tabular}{cccc}
\hline Parameters & Variation & Input Power (Watt) & $\Delta$ Tel (K) \\
\hline Frequency & from 1 to 60 kHz & For any given & $<10$ \\
Electrode area & from 3 to $1 \mathrm{~cm}^{2}$ & 13 & +20 \\
Gas flow rate & from 1 to 6 L.min & 14 & -50 \\
\multirow{2}{*}{ Heat exchanges } & Limited (thermal cover) & 12 & +30 \\
\cline { 2 - 4 } & Forced (fan) & 12 & -20 \\
\hline
\end{tabular}

As already shown in section 3.2, for a given input power, the electrode temperature is constant for all tested frequencies. Decoupling temperature from electrical parameters in DBD 
is possible by controlling thermal exchanges through the electrode shape, the gas flow rate, and the thermal conditioning of the DBD reactor by modifying heat exchange around the casings (with either insulation or a fan).

Heat exchange around the electrode depends on its shape and its thermal conductivity. The reduction of electrode area at constant input power leads to an increase of dielectric surface temperature. For instance at 13 watt, electrode temperature increases by $20 \mathrm{~K}$ for a factor 3 on the number of filament per surface unit. This effect is due to the increase of the number of filaments per surface unit and per period, as well as the reduction of radial heat exchange on the metal electrode.

The modification of heat exchange around the DBD casing either by limiting or forcing the convection allows the control of the dielectric surface temperature at constant input power (e.g. at 12 Watts per $3 \mathrm{~cm}^{2}$, the dielectric surface temperature is $300 \mathrm{~K}$ and is tuneable from 280 to $330 \mathrm{~K}$ ). For the same input power and the same distribution of the filaments in time and space, the thermal conditioning of the DBD reactor leads to tuneable temperature within $50 \mathrm{~K}$.

In most industrial processes based on DBD, accurate control of heat and mass transfer and kinetics is required. As the temperature profile depends not only on plasma properties but also on external cooling, the reactor design is thus important. Depending on the application, the discharge gap, the size of the discharge and the reactor as well as the operating conditions can be chosen to define the temperature range of the DBD. The key parameters governing the heat source (filaments) are the input power and the average surface input power. The key parameters governing the heat sink are the external surface of the reactor, the ambient temperature as well as the mass flow rate and the initial temperature of flowing gas in the DBD. The reduction of reactor size, for space requirement, often imposes the use of additional cooling systems to control the optimal temperature range.

However, the control of average gas and surface temperatures is not sufficient to control the transport of chemical species and aerosol particles. The temperature profiles around each filament have to be considered to account for transport phenomena (diffusion, convection and thermophoresis). Thus, the following section is devoted to local temperature gradients around filamentary discharges.

\subsection{Plasma temperature and local temperature gradient}

The aim of this section is to show that the thermal profile can be tuned by varying the applied voltage frequency.

Without any gas flow in the reactor, the evolution of mean gas temperature is evaluated from electrode temperature. Temperature of neutral gas molecules in the plasma channel (here after referred as plasma temperature), is evaluated as described in the experimental setup. This evaluation overestimates the plasma temperature in the filament due to the collection of the light from the spots situated at the filament bases. Figure 5 (a) represents the evolution of mean gas temperature and plasma temperature in the $1 \mathrm{~mm}$ gap width DBD reactor, at $60 \mathrm{kHz}$ versus the input power (i.e. versus the number of filaments per half period and per square centimetre).

The average difference of temperature between the plasma and the surrounding gas, referred as the temperature rise in the filament is plotted versus the voltage frequency in Figure 5 (b). 

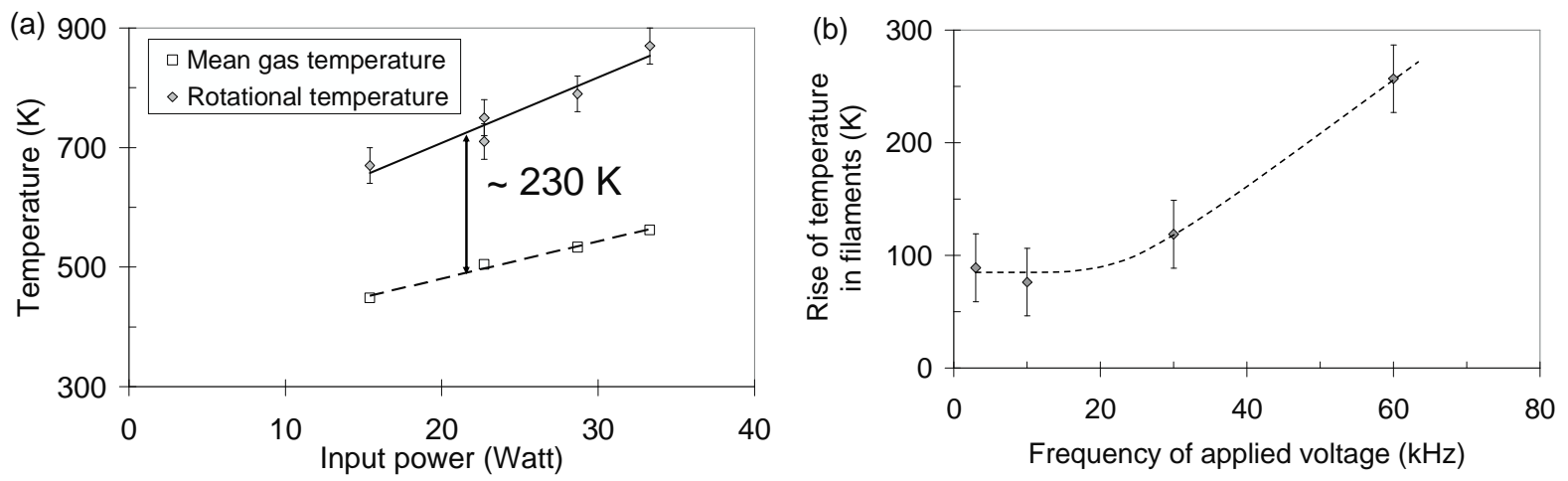

Figure 5: (a) Mean temperatures of gas and plasma versus input power $(60 \mathrm{kHz})$

(b) Temperature rise in the filament versus frequency.

At $60 \mathrm{kHz}$, the plasma temperature is about $230 \mathrm{~K}$ higher than the surrounding gas temperature (cf. Figure 5 a), no matter what the number of filaments per surface and time unit is. This result is confirmed during the initial warm-up of the reactor, from ambient temperature to thermal equilibrium, during which the same temperature difference between the filament and the bulk gas is measured. For a given voltage frequency, the difference of temperature between plasma temperature and surrounding gas is constant. Thus, for a given energy per filament and voltage frequency, the local gradients around a filament are constant leading to similar transport phenomena around each filament.

The thermal gradients around a filament can be controlled either by the energy per filament or by the frequency of the applied voltage. The average temperature difference between the filament and surrounding gas increases with increasing energy per filament and frequency. If an adiabatic expansion of the filament channel occurs after the filament propagation [14], then the modification of temperature gradient can be smoothed.

With a $0.5 \mathrm{~mm}$ gap, the energy per filament is half smaller than with $1 \mathrm{~mm}$ gap and the temperature rise in the filament is reduced from $230 \mathrm{~K}$ to $100 \mathrm{~K}$, as already observed in [32].

In Figure 5 (b), for frequencies the below $10 \mathrm{kHz}$, the temperature rise is constant at about $80 \mathrm{~K}$ (cf. Figure $5 \mathrm{~b}$ ). For the frequencies above $10 \mathrm{kHz}$, the temperature rise in the filament increases with the frequency. This effect has already been reported in $\mathrm{N}_{2} / \mathrm{Ar}$ at 100 Torr where filament temperature increased from 380 to $400 \mathrm{~K}$ with the frequency from $0.5 \mathrm{kHz}$ to $30 \mathrm{kHz}$ [33]. The residual heating of the filament channel between two successive filaments occurring at the same location in successive half periods is involved. Hence, the relaxation time constant of temperature can be estimated at about $0.1 \mathrm{~ms}$ in dry air at atmospheric pressure, which is consistent with [14, 13].

Above $15 \mathrm{kHz}$, the residual heating of the filament channel leads to a lower onset voltage for filament development. As filaments have similar charge per pulse (cf. section 3.1) the reduction of onset voltage should lead to a reduction of the energy per filament. This is confirmed in Figure 6 which represents the mean surface energy density per period (the input power divided by the frequency and the electrode surface area) versus the peak-to-peak applied voltage for the $0.5 \mathrm{~mm}$ gap DBD. 


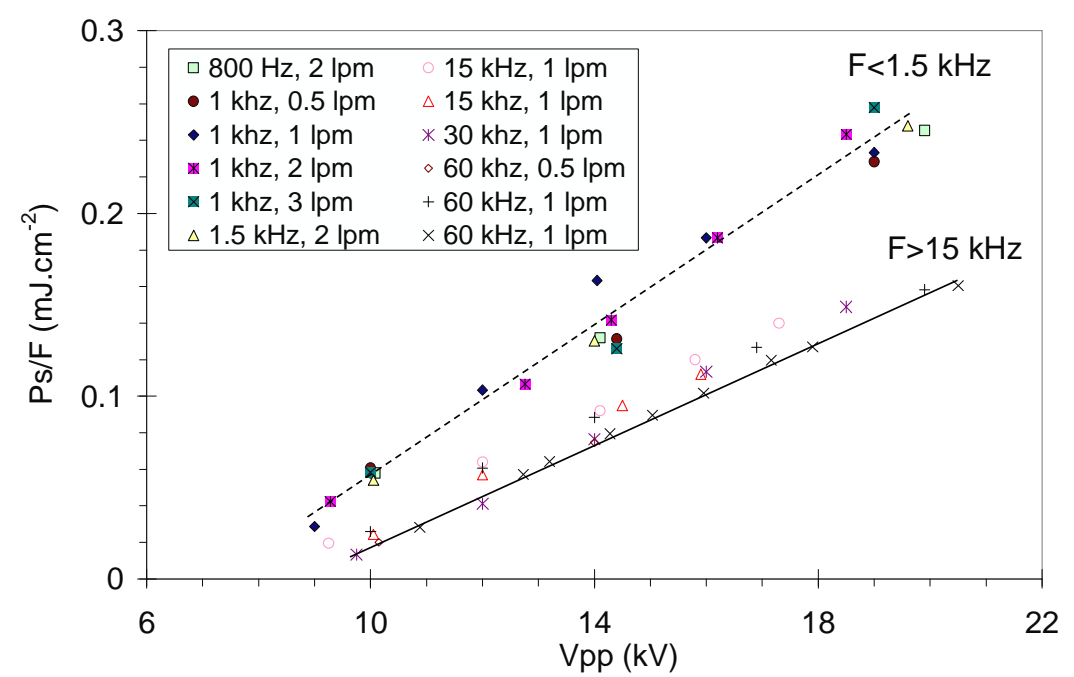

Figure 6: Mean surface energy density per period at different frequencies from 0.8 to $60 \mathrm{kHz}, 0.5 \mathrm{~mm}$ gap.

For a given frequency, the mean surface energy density injected by period (Ps/F in J.m ${ }^{-2}$, with Ps, the ratio of input power and surface electrode, and $F$ the frequency) increases linearly with the applied voltage due to increasing number of similar filaments per surface unit and per period. For a given voltage, the energy per period is constant from 0.8 to $1.5 \mathrm{kHz}$ and is lower for frequencies above $15 \mathrm{kHz}$. This reduction of energy is due to the increase of the gas temperature in which the filament develops. This is confirmed by the reduction of the measured input power while the gas temperature increases at $60 \mathrm{kHz}$ for a given voltage.

Assuming a constant number of filaments per period, the reduction of energy per period is related to the reduction of the energy per filament. As the charge per filament is constant (cf. section 3.1), the reduction of the energy per filament is due to the reduction of the onset voltage of the filament. At $20 \mathrm{kV}$, the energy per period is $0.72 \mathrm{~mJ}$ at $1 \mathrm{kHz}$ and $0.47 \mathrm{~mJ}$ at $60 \mathrm{kHz}$. Assuming a constant reduced field threshold for the formation of the filament, the ratio of filament energy is the inverse of temperature ratio. As the gas temperature is around $300 \mathrm{~K}$ at $1 \mathrm{kHz}$, the gas temperature at $60 \mathrm{kHz}$ should thus be around $459 \mathrm{~K}(0.72 / 0.47 * 300$ $\mathrm{K})$. In fact, the average gas temperature is about $435 \mathrm{~K}$, the difference being due to filament channel residual heating.

To summarize, the reduction of energy per period with increasing frequency is due to the reduction of the energy per filament. The lower energy per filament is due to lower onset voltage at constant charge per filament, related to both increasing average gas temperature and residual heating of the filament channel for frequency higher than $10 \mathrm{kHz}$.

\section{Conclusions}

This paper presents a method to identify the influences of gas and surface temperatures as well as temperature gradient on the relative importance of different physical and chemical mechanisms at constant filamentary discharge electrical characteristics. The main regulating parameters to control temperature and thermal gradient are depicted.

For given operating conditions, the mean dielectric surface temperature linearly increases with the input power density and is independent of the dynamics of energy injection (energy per filament and filament distribution in time and space).

Gas flow rate and thermal conditioning (forced or limited convection around the reactor) allow the modification of the equilibrium temperatures without changing the electrical parameters. 
The invariance of the temperature rise in the filament, for a given discharge arrangement at a given frequency, no matter what the surrounding gas temperature is, as well as the increase of the temperature rise in the filament with the energy per filament have been confirmed.

Moreover, it was shown that the temperature rise is constant below $1.5 \mathrm{kHz}$ ( $80 \mathrm{~K}$ with a 1 mm gap DBD) but increases for frequencies higher than $15 \mathrm{kHz}$ (up to $230 \mathrm{~K}$ with a $1 \mathrm{~mm}$ gap DBD, at $60 \mathrm{kHz}$ ) due to residual heat from the preceding one at the same location. The temperature gradient around filaments can thus be controlled by the frequency of the applied voltage above $15 \mathrm{kHz}$.

Based on these results, the respective influences of transport (diffusion, convection and thermophoresis) and kinetics (chemical, nucleation) involved in the production of chemical species and aerosol may be determined by controlling radial thermal gradients around filamentary discharges as well as gas and surface temperatures with constant filament electrical characteristics (charge per filament, reduced electric field and electron energy distribution function).

These results are of particular interest when DBD is used for specific chemical reaction and especially for thermal conditioning of catalysts or for thin film deposition processes by chemical vapour deposition. Moreover, the kinematics of aerosols injected or produced in DBD depends on the thermal profile of the DBD and has to be controlled to develop aerosol processes based on DBD.

Acknowledgments

The authors wish to thank Dr. Robert Haug for his contribution in thermal simulations as well as Dr. Michael Kirkpatrick and Dr. Syed Salman Asad for English corrections.

References

[1] Kogelschatz U, Eliasson B and Egli W 1999 From ozone generators to flat television screens: history and future potential of dielectric-barrier discharges. In: 14th International Symposium on Plasma Chemistry, (Prague, Czech Republic: Int Union Pure Applied Chemistry) pp 1819-28

[2] Kogelschatz U 2003 Dielectric-barrier discharges: Their history, discharge physics, and industrial applications Plasma Chem. Plasma Process. 23 1-46

[3] Kozlov K V, Wagner H E, Brandenburg R and Michel P 2001 Spatio-temporally resolved spectroscopic diagnostics of the barrier discharge in air at atmospheric pressure Journal of Physics D-Applied Physics 34 3164-76

[4] Hammer T 1999 Application of plasma technology in environmental techniques Contrib. Plasma Phys. 39 441-62

[5] Fridman A, Chirokov A and Gutsol A 2005 Non-thermal atmospheric pressure discharges Journal of Physics D-Applied Physics 38 R1-R24

[6] Kogelschatz U, Eliasson B and Egli W 1997 Dielectric-barrier discharges. Principle and applications. In: XXIIIrd International Conference on Phenomena in Ionized Gases, (Toulouse, France: E D P Sciences) pp 47-66

[7] Massines F, Rabehi A, Decomps P, Gadri R B, Segur P and Mayoux C 1998 Experimental and theoretical study of a glow discharge at atmospheric pressure controlled by dielectric barrier J. Appl. Phys. 83 2950-7

[8] Rahel J, Sira M, Stahel P and Trunec D 2007 The transition between different discharge regimes in atmospheric pressure air barrier discharge Contrib. Plasma Phys. 47 34-9

[9] Eliasson B, Hirth M and Kogelschatz U 1987 Ozone synthesis from oxygen in dielectric barrier discharges Journal of Physics D-Applied Physics 20 1421-37

[10] Borra J P 2006 Nucleation and aerosol processing in atmospheric pressure electrical discharges: powders production, coatings and filtration Journal of Physics D-Applied Physics 39 R19-R54

[11] Borra J P, Jidenko N and Bourgeois E 2009 Atmospheric pressure plasmas for aerosols processes in materials and environment The European Physical Journal Applied Physics 4722804

[12] Parissi L, Goldman A, Goldman M, Odic E and Borra J P 1999 Electrical discharges for environmental purposes: fundamentals and applications, ed E M v Veldhuizen (New York: NOVA Science Publishers) pp 279-312 
[13] Eichwald O, Guntoro N A, Yousfi M and Benhenni M 2002 Chemical kinetics with electrical and gas dynamics modelization for NOx removal in an air corona discharge Journal of Physics D-Applied Physics 35 439-50

[14] Marode E, Samson S, Djermoune D, Deschamps N, Touzeau M and De Souza R 1999 Time Resolved Temperature Measurements and Computation of Streamer Air Discharge and Diffusion Controled Chemistry Journal of Advanced Oxidation Technologies 4 305-11

[15] Andrews T and Tait P G 1861 On the volumetric relations of Ozone and the action of the electrical discharge on oxygen and other gases $Q$. J. Chem. Soc. 13 344-67

[16] Borra J P 2008 Charging of aerosol and nucleation in atmospheric pressure electrical discharges Plasma Physics and Controlled Fusion 124036

[17] Jidenko N and Borra J P 2005 Kinematics of charged nanometric particles in silent discharges J. Phys D.: Apply Phys $\mathbf{3 8} 617$

[18] Motret O, Hibert C, Pellerin S and Pouvesle J M 2000 Rotational temperature measurements in atmospheric pulsed dielectric barrier discharge - gas temperature and molecular fraction effects Journal of Physics D-Applied Physics 33 1493-8

[19] Nozaki T, Miyazaki Y, Unno Y and Okazaki K 2001 Energy distribution and heat transfer mechanisms in atmospheric pressure non-equilibrium plasmas Journal of Physics D: Applied Physics 34 3383-90

[20] Kappesa T, Schiene W and Hammer T 2002 Energy balance of a dielectric barrier discharge reactor for hydrocarbon stream reforming. In: Hakone8 (Estonia

[21] Sadat H, Dubus N, Pinard L, Tatibouet J M and Barrault J 2009 Conduction heat transfer in a cylindrical dielectric barrier discharge reactor Applied Thermal Engineering 29 1259-63

[22] Xu X D P and Kushner M J 1998 Ion composition of expanding microdischarges in dielectric barrier discharges J. Appl. Phys. 83 7522-32

[23] Jidenko N, Petit M and Borra J P 2006 Electrical characterization of microdischarges produced by dielectric barrier discharge in dry air at atmospheric pressure J. Phys D.: Apply Phys 39281

[24] Chelouah A, Marode E, Hartmann G and Achat S 1994 A new method for temperature evaluation in a nitrogen discharge Journal of Physics D-Applied Physics 27 940-5

[25] Hartmann G and Johnson P C 1978 Measurements of relative transition-probabilties and variation of electronic-transition moment for N2 C PI-3(U)-B PI-3(G) second positive system J. Phys. B-At. Mol. Opt. Phys. 11 1597-612

[26] Petit M, Jidenko N, Goldman A, Goldman M and Borra J P 2002 Electrical characterization of gas discharges using a numerical treatment. Application to dielectric barrier discharges Review of Scientific Instruments 73 2705-12

[27] Fridman A 2008 Plasma Chemistry (Cambridge: Cambridge University Press)

[28] Kogelschatz U 1983 Ozone synthesis in gas discharge. In: International Conference on Phenomena in Ionized Gases 16, (Dusseldorf) pp 240-50

[29] Gibalov V I and Pietsch G J 2000 The development of dielectric barrier discharges in gas gaps and on surfaces Journal of Physics D-Applied Physics 33 2618-36

[30] Bourgeois E, Jidenko N, Alonso M and Borra J P 2009 DBD as a post-discharge bipolar ions source and selective ion-induced nucleation versus ions polarity Journal of Physics D-Applied Physics 42

[31] Kappes T, Schiene W and Hammer T 2002 Energy balance of a dielectric barrier discharge reactor for hydrocarbon stream reforming. In: Hakone8 (Estonia

[32] Braun D, Gibalov V and Pietsch G 1992 Two-dimensional modelling of the dielectric barrier discharge in air Plasma Sources Science and Technology 166

[33] Williamson J M, Bletzinger P and Ganguly B N 2004 Gas temperature determination in a N2/Ar dielectric barrier discharge by diode-laser absorption spectroscopy and resolved plasma emission Journal of Physics D: Applied Physics 1658 